\title{
An overnight comparison of two ventilators used in the treatment of chronic respiratory failure
}

\author{
M.P. Highcock, E. Morrish, S. Jamieson, J.M. Shneerson, I.E. Smith
}

\begin{abstract}
An overnight comparison of two ventilators used in the treatment of chronic respiratory failure. M.P. Highcock, E. Morrish, S. Jamieson, J.M. Shneerson, I.E. Smith. (C)ERS Journals Ltd 2002.

ABSTRACT: Differences between bilevel ventilators used for noninvasive intermittent positive pressure ventilation (NIPPV) have been demonstrated during bench testing. However, there are no clinical studies comparing these machines. The authors have previously shown that the Quantum pressure support ventilator and Sullivan variable positive airway pressure II ST differ in performance during bench testing. To examine the clinical significance of this, these two machines were compared in the overnight treatment of subjects with chronic respiratory failure.

Ten clinically-stable subjects with thoracic scoliosis were recruited. The subjects were already established on NIPPV, but none were using either of the ventilators to be tested. After familiarisation, the patients used the two ventilators in random order on consecutive nights. Peripheral oxygen saturation and transcutaneous carbon dioxide tension $\left(\mathrm{P}_{\mathrm{t}}, \mathrm{CO}_{2}\right)$ were measured continuously, and sleep was recorded using polysomnography.

There were no significant differences in arterial oxygen saturation, $\mathrm{Pt}_{\mathrm{t}} \mathrm{CO}_{2}$ or sleep duration and quality between the two nights.

Despite previously illustrated variation in laboratory performance, no differences were seen between the two ventilators when comparing overnight gas exchange and sleep in vivo. Further study is required to evaluate the significance of the differences found during bench testing in the clinical setting.

Eur Respir J 2002; 20: 942-945.
\end{abstract}

The Respiratory Support and Sleep Centre, Papworth Hospital, Papworth Everard, Cambridge, UK.

Correspondence: M.P. Highcock

The Respiratory Support and Sleep Centre

Papworth Hospital

Papworth Everard

Cambridge

CB3 8RE

UK

Fax: 441480830620

E-mail: martin.highcock@dbh.nhs.uk

Keywords: Bilevel pressure ventilators chronic respiratory failure

nocturnal ventilation

sleep quality

thoracic scoliosis

Received: November 292001

Accepted after revision: May 222002
Noninvasive positive pressure ventilation (NIPPV) is well established in the treatment of chronic respiratory failure. Regular nocturnal treatment improves overnight oxygen saturation and daytime arterial blood gases in obstructive and restrictive pulmonary disorders $[1,2]$. There are beneficial effects on sleep duration and efficiency and improvements in quality of life [3, 4]. The outlook is particularly favourable in patients with chest wall or nonprogressive neuromuscular diseases, with almost $80 \%$ of these patients continuing to benefit from treatment after 5 yrs [5].

Despite the success of treatment, the optimal mode of ventilation is not known. Pressure preset ventilation is increasingly popular, but its superiority over volume preset ventilation is unproven. There are no differences between these two modes when comparing the improvements seen in gas exchange in patients with chronic $[6,7]$ or acute $[8]$ respiratory failure. It has been argued that pressure preset ventilation has the advantage of leak compensation $[9,10]$, and some patients who fail on a volume preset device may benefit from changing the ventilator [11]. However, in a study of patients well treated on a volume preset ventilator, a proportion deteriorated when tried on pressure preset ventilation [12].

There are no clinical studies comparing pressure preset ventilators, although laboratory comparisons of such devices have shown differences between the machines tested $[13,14]$. The authors have previously shown that the Quantum pressure support ventilator (PSV) (Healthdyne/Respironics Inc., Murrysville, PA, USA) and Sullivan variable positive airway pressure (VPAP) II ST (Resmed Ltd, North Ryde, Australia) differ in performance during bench testing [15]. To examine the clinical significance of this these two machines were compared in the overnight treatment of stable subjects with chronic respiratory failure due to thoracic scoliosis.

\section{Methods}

Subjects were recruited from the population of patients attending the authors' unit with respiratory failure due to thoracic scoliosis. Those already established on home nocturnal NIPPV for $\geqslant 6$ months were eligible. Subjects were excluded if they required supplemental oxygen, were already using either of the two ventilators to be studied or had any change of symptoms or drug therapy in the 4 weeks prior to the study. All subjects gave informed consent.

Two ventilators, Quantum PSV (Healthdyne/ Respironics Inc.) and Sullivan VPAP II ST (Resmed 
Ltd), were compared. They were used in the spontaneous/timed mode with the minimum expiratory pressure. During a period of familiarisation, the subjects selected all the other settings for each machine. A preference for peak inspiratory positive airway pressure (IPAP), risetime and respiratory rate were noted independently according to comfort. The subjects used their own mask and the same circuit incorporating a whisper swivel II expiratory valve (Respironics Inc.) with each ventilator.

On the first day of attendance, the inclusion and exclusion criteria were reviewed and baseline measurements were recorded. Spirometry was performed using a rolling seal spirometer (Vitalograph Ltd, Buckingham, UK) and total lung capacity (TLC) was estimated using body plethysmography (Masterlab, Jaeger, Germany). Gas transfer could not be measured due to low intrathoracic gas volumes. A penetrated posteroanterior chest radiograph was performed so that the Cobb angle could be calculated. Arterial blood gas (ABG) tensions were measured at rest, breathing room air.

Overnight studies were then performed on consecutive nights, using each of the two ventilators in random order. A period of familiarisation during the day preceded the night-time study. Peripheral oxygen saturation $\left(\mathrm{Sp}, \mathrm{O}_{2}\right)$ using a finger probe (Datex Ohmeda, Helsinki, Finland) and transcutaneous carbon dioxide tensions $\left(P \mathrm{t}, \mathrm{CO}_{2}\right)$ using a heated skin electrode (TINA, Radiometer, Copenhagen, Denmark) were monitored during familiarisation to ensure that the ventilator settings were optimal.

During each night of the study a number of measurements were made. Standard electroencephalogram, electro-oculogram and submental electro-myogram were recorded onto a data-logging system (Alice 3, Healthdyne/Respironics Inc.). The recordings were used to stage sleep by two experienced polysomnographers who were blinded as to which ventilator was used on which night. $\mathrm{Sp}, \mathrm{O}_{2}$ and $\mathrm{Pt}_{\mathrm{t}} \mathrm{CO}_{2}$ were recorded continuously onto a second datalogging system (CARDAS, Oxcams Medical Sciences Ltd, Oxford, UK). The $P \mathrm{t}, \mathrm{CO}_{2}$ signal was calibrated against an $\mathrm{ABG}$ specimen on each night.

\section{Statistics}

Data are presented as mean \pm SD. Paired t-tests were used to compare parameters and a p-value of $<0.05$ was considered significant.

\section{Results}

Ten subjects (three females) were recruited. One had an idiopathic adolescent scoliosis, but all the others had a congenital scoliosis. The spinal deformity was severe with a Cobb angle $101 \pm 20.4$ degrees, forced expiratory volume in one second $0.7 \pm 0.3 \mathrm{~L}(24 \%$ predicted), forced vital capacity $1.1 \pm 0.3 \mathrm{~L}$ ( $27 \%$ pred), TLC $2.5 \pm 0.56 \mathrm{~L}(39 \%$ pred). Subjects were well established on mask ventilation (mean 54.7, range 11-120 months) and ABG tensions confirmed that they were adequately treated; oxygen tension in arterial blood $10.4 \pm 0.8 \mathrm{kPa}$, carbon dioxide tension in arterial blood $6.0 \pm 0.4 \mathrm{kPa}$.

The settings on the two test ventilators were compared. There was no difference in: the preset IPAP (Quantum 21.0 $\pm 3.5 \mathrm{cmH}_{2} \mathrm{O}$ and VPAP 20.8土 $3.6 \mathrm{cmH}_{2} \mathrm{O}, \mathrm{p}=0.69$ ); the preset risetime (Quantum $0.5 \pm 0.4 \mathrm{~s}$ and VPAP $0.3 \pm 0.0 \mathrm{~s}, \mathrm{p}=0.14)$; and the preset respiratory rate (Quantum $15.4 \pm 1.2$ breaths per minute (bpm) and VPAP $15.0 \pm 1.5 \mathrm{bpm})$. Three subjects volunteered an overall preference for the Quantum and five favoured the VPAP.

\section{Sleep data}

For three of the nights, from two subjects (nos. 7 and 10), it was not possible to score sleep due to the poor quality of the record. From the complete data (eight subjects), no differences were seen between the nights with each ventilator for total sleep time, sleep latency, sleep efficiency (total sleep time/time from first sleep page to last sleep page) or arousals (table 1). Similarly, there were no significant differences in the durations of light (stage 1 and 2) and deep (stage 3 and 4) nonrapid eye movement or rapid eye movement sleep (fig. 1). A number of subjects commented that they found it more difficult to sleep than normal due to the monitoring equipment used.

Table 1.-Comparative sleep data

\begin{tabular}{lccc}
\hline & Quantum & VPAP & p-value \\
\hline Total sleep time min & $303.2 \pm 79.4$ & $314.4 \pm 54.2$ & 0.72 \\
Sleep latency min & $20.1 \pm 14.4$ & $21.8 \pm 13.5$ & 0.21 \\
Sleep efficiency \% & $73.1 \pm 17.4$ & $79.8 \pm 10.1$ & 0.23 \\
${\text { Arousal index } \mathrm{h}^{-1}}^{7.7 \pm 4.7}$ & $8.0 \pm 5.3$ & 0.85 \\
\hline
\end{tabular}

Data are presented as mean \pm SD. VPAP: variable positive airway pressure II ST; Quantum: Quantum pressure support ventilator. Manufacturer's details are as follows: Quantum (Healthdyne/Respironics Inc., Murrysville, PA, USA), VPAP (Resmed Ltd, North Ryde, Australia).

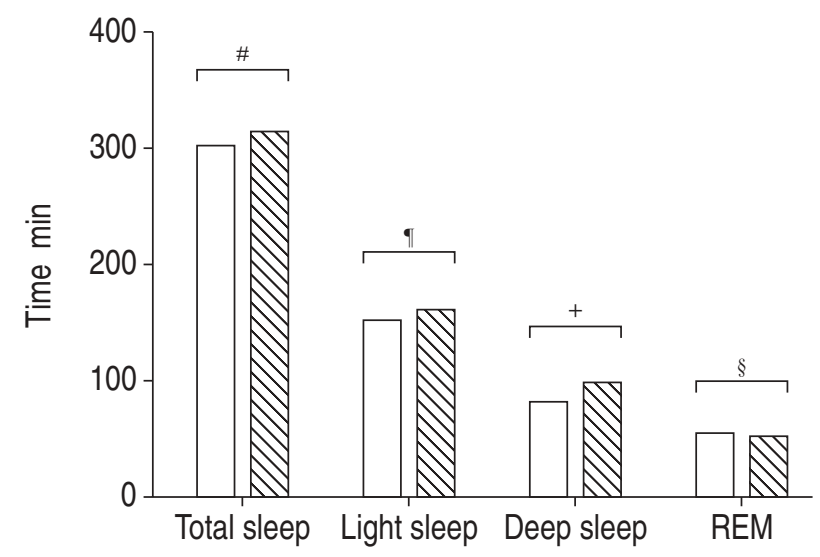

Fig. 1.-Duration of sleep (total, light, deep and nonrapid eye movement (REM)) in subjects using the Quantum pressure support ventilator $(\square)$ and the Sullivan variable positive airway

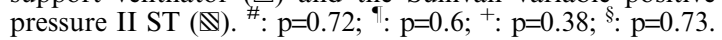


Table 2.-Comparitive oximetry and transcutaneous carbon dioxide tension $\left(\mathrm{Pt}, \mathrm{CO}_{2}\right)$ data

\begin{tabular}{lclc}
\hline & Quantum & VPAP & p-value \\
\hline Mean $S \mathrm{p}, \mathrm{O}_{2} \%$ & $92.5 \pm 2.6$ & $92.8 \pm 2.1$ & 0.46 \\
Minimum $S_{\mathrm{p}, \mathrm{O}_{2}} \%$ & $81.5 \pm 15.3$ & $87.5 \pm 1.9$ & 0.24 \\
$S_{\mathrm{p}, \mathrm{O}_{2}<90 \% \text { min }}<9.4 \pm 122.1$ & $56.0 \pm 103.8$ & 0.33 \\
$S_{\mathrm{p}, \mathrm{O}_{2}<90 \% \text { no. }}<$ & $23.9 \pm 31.3$ & $22.3 \pm 26.2$ & 0.89 \\
$4 \%$ desaturations no. & $8.8 \pm 5.0$ & $9.8 \pm 6.6$ & 0.42 \\
Mean $P \mathrm{t}, \mathrm{CO}_{2} \mathrm{kPa}$ & $7.3 \pm 1.3$ & $7.1 \pm 1.2$ & 0.52 \\
\hline
\end{tabular}

Data are presented as mean \pm SD. VPAP: variable positive airway pressure II ST; Quantum: Quantum pressure support ventilator; $S \mathrm{p}, \mathrm{O}_{2}$ : peripheral oxygen saturation; $\mathrm{Pt}_{\mathrm{t}, \mathrm{CO}_{2}}$ : transcutaneous carbon dioxide tension. Manufacturer's details are as follows: Quantum (Healthdyne/Respironics Inc., Murrysville, PA, USA), VPAP (Resmed Ltd, North Ryde, Australia).

\section{Oximetry and transcutaneous carbon dioxide tension}

For five nights, from three subjects (nos. 4, 5 and 7), the $\mathrm{Pt}, \mathrm{CO}_{2}$ record could not be used because of the poor quality of the signal. Comparing the complete data, there were no significant differences in $\mathrm{Pt}_{\mathrm{t}} \mathrm{CO}_{2}$ (seven subjects) or $S_{\mathrm{p}, \mathrm{O}_{2}}$ (10 subjects) between the two nights (table 2).

\section{Discussion}

The authors have previously shown that the Quantum and VPAP differ in performance during bench tests in a number of respects [15]. Both ventilators compensate well when a leak is introduced into the circuit, with any fall in the resulting tidal volumes $(V T)$ being $<10 \%$. Inspiratory time with the VPAP, however, is prolonged by the presence of a leak. This leads to an increase in the $V \mathrm{~T}$, but theoretically could lead to incoordination and difficulty with expiration at rapid respiratory rates. The trigger for the VPAP is more sensitive than that of the Quantum, and thus, less patient work is expended in triggering the ventilator. In addition, there were unexpected effects on $V \mathrm{~T}$ when the ventilators were triggered by simulated patient effort. $V \mathrm{~T}$ with the Quantum fell due to premature expiratory cycling. In contrast, simulated patient effort was "rewarded" by the VPAP with an increase in the resulting $V \mathrm{~T}$ during some tests.

Therefore, there are a number of differences between the machines that could particularly influence the interaction between the patient and the ventilator. Despite the differences seen during bench testing, there were no differences in the overnight treatment of stable patients with chronic respiratory failure due to thoracic scoliosis.

This may be because there is a real difference between the two machines that was not detected due to insufficient patient numbers. The total number of subjects included in the present study was small. However, the results are closely matched and there is no suggestion of a trend toward one or other of the ventilators from the data. If a large number of subjects are required to demonstrate a statistically significant difference, then the clinical significance is likely to be small.

The relevance of bench tests to the clinical situation is uncertain. Bench testing is sensitive to small differences between devices. All published studies have shown statistically significant results [9, 13-15], while several clinical studies comparing machines and modes of ventilation have shown no differences in outcome [6-8, 16, 17]. The differences measured on bench tests may not be clinically relevant or patients may be able to compensate for them. LofAso et al. [13] showed differences between two ventilators in trigger sensitivity, initial flow rate and imposed work on bench tests. When the same two devices were compared in a clinical trial with intubated patients, the subjects increased their work of breathing to maintain equivalent ventilation and gas exchange when using the ventilator shown to be inferior in the bench tests. The clinical outcome may be affected if a higher work of breathing is imposed in the long-term, particularly in subjects liable to fatigue, but this has not so far been demonstrated.

Some subjects deteriorate despite regular NIPPV and subsequently improve when the ventilator is changed [11]. The precise reason for this improvement is not known, but may relate to differences in the ventilators that are apparent on bench testing [9]. It is probable that differences in devices illustrated in the laboratory have some clinical relevance, but the outcome will vary according to the precise clinical situation.

NIPPV is particularly well established in the treatment of subjects with chest wall disorders [2] and the long-term prognosis is good [5, 18]. The outcome of regular NIPPV in obstructive lung disease has improved in recent years, but remains less favourable $[1,5]$. NIPPV improves outcome in acute respiratory failure $[19,20]$ and acute exacerbations of chronic obstructive pulmonary disease [21, 22]. However, 15-31 per cent of patients still require intubation [19-22] and mortality varies between 6-38\% [19-22]. Subjects were taken from a group that respond well to treatment and were clinically stable. Small variations in ventilator performance might not be detectable over a single night of treatment. A longer period of time may be required to translate small differences in work of breathing and ventilation into worsening gas exchange and sleep disturbance. It is also probable that differences would be more apparent in patient groups that were more difficult to treat, such as those with obstructive lung disease or acute respiratory failure.

In conclusion, there is a paucity of data on which to inform decisions regarding selection of ventilators for noninvasive intermittent positive pressure ventilation. Bench tests have illustrated differences between devices $[9,13-15]$, but the clinical significance of these findings remains uncertain. In comparing pressure and volume preset ventilation, differences in clinical outcome may only become apparent during long-term treatment $[11,12]$. There are no long-term studies comparing bilevel positive pressure ventilators. The present authors did not illustrate any difference between the two machines tested. This may have 
been due to insufficient patient numbers, too short a treatment period or a patient group that was relatively easy to ventilate. This was in effect a pilot study. The ideal design for a future study would be a randomised controlled clinical trial comparing the outcome of two or more devices used during the initial treatment period in subjects presenting for the first time with respiratory failure.

\section{References}

1. Sivasothy P, Smith IE, Shneerson JM. Mask intermittent positive pressure ventilation in chronic hypercapnic respiratory failure due to chronic obstructive pulmonary disease. Eur Respir J 1998; 11: $34-40$.

2. Bach JR, Alba AS. Management of chronic alveolar hypoventilation by nasal ventilation. Chest 1990; 97 : 52-57.

3. Elliott MW, Simonds AK, Carroll MP, Wedzicha JA, Branthwaite MA. Domiciliary nocturnal nasal intermittent positive pressure ventilation in hypercapnic respiratory failure due to chronic obstructive lung disease: effects on sleep and quality of life. Thorax 1992; 47: 342-348.

4. Meecham Jones DJ, Paul EA, Jones PW, Wedzicha JA. Nasal pressure support ventilation plus oxygen compared with oxygen alone in hypercapnic COPD. Am J Respir Crit Care Med 1995; 152: 538-544.

5. Simonds AK, Elliott MW. Outcome of domiciliary nasal intermittent positive pressure ventilation in restrictive and obstructive disorders. Thorax 1995; 50: 604-609.

6. Meecham Jones DJ, Wedzicha JA. Comparison of pressure and volume preset nasal ventilator systems in stable chronic respiratory failure. Eur Respir J 1993; 6: 1060-1064.

7. Elliott MW, Aquilina R, Green M, Moxham J, Simonds AK. A comparison of different modes of non-invasive ventilatory support: effects on ventilation and inspiratory muscle effort. Anaesthesia 1994; 49: 279-283.

8. Meecham Jones DJ, Paul EA, Grahame-Clarke C, Wedzicha JA. Nasal ventilation in acute exacerbations of chronic obstructive pulmonary disease: effect of ventilator mode on arterial blood gas tensions. Thorax 1994; 49: 1222-1224.

9. Smith IE, Shneerson JM. A laboratory comparison of four positive pressure ventilators used in the home. Eur Respir J 1996; 9: 2410-2415.

10. Mehta S, McCool FD, Hill NS. Leak compensation in positive pressure ventilators: a lung model study. Eur Respir J 2001; 17: 259-267.

11. Smith IE, Shneerson JM. Secondary failure of nasal intermittent positive pressure ventilation using the Monnal D: effects of changing the ventilator. Thorax 1997; 52: 89-91.

12. Schonhofer B, Sonneborn M, Haidl P, Bohrer H, Kohler D. Comparison of two different modes of noninvasive mechanical ventilation in chronic respiratory failure: volume versus pressure controlled device. Eur Respir J 1997; 10: 184-191.

13. Lofaso F, Brochard L, Hang T, Lorino H, Harf A, Isabey D. Home versus intensive care pressure support devices: experimental and clinical comparison. $\mathrm{Am}$ J Respir Crit Care Med 1996; 153: 1591-1599.

14. Bunburaphong $\mathrm{T}$, Imanaka $\mathrm{H}$, Nishimura M, Hess D, Kacmarek RM. Performance characteristics of bilevel pressure ventilators. Chest 1997; 111: 1050-1060.

15. Highcock MP, Shneerson JM, Smith IE. Functional differences in bi-level pressure pre-set ventilators. Eur Respir J 2001; 17: 268-273.

16. Vitacca M, Rubini F, Foglio K, Scalvini S, Nava S, Ambrosino N. Non-invasive modalities of positive pressure ventilation improve the outcome on acute exacerbations in COLD patients. Intensive Care Med 1993; 19: 450-455.

17. Restrick LJ, Fox NC, Braid G, Ward EM, Paul EA, Wedzicha JA. Comparison of nasal pressure support ventilation with nasal intermittent positive pressure ventilation in patients with nocturnal hypoventilation. Eur Respir J 1993; 6: 364-370.

18. Baydur A, Layne E, Aral H, et al. Long term noninvasive ventilation in the community for patients with musculoskeletal disorders: 46 years experience and review. Thorax 2000; 55: 4-11.

19. Kramer N, Meyer TJ, Meharg J, Cece RD, Hill NS. Randomized, prospective trial of noninvasive positive pressure ventilation in acute respiratory failure. $\mathrm{Am}$ J Respir Crit Care Med 1995; 151: 1799-1806.

20. Antonelli M, Conti G, Rocco M, et al. A Comparison of noninvasive positive-pressure ventilation and conventional mechanical ventilation in patients with acute respiratory failure. $N$ Eng $J$ Med 1998; 339: 429-435.

21. Brochard L, Mancebo J, Wysocki M, et al. Noninvasive ventilation for acute exacerbations of chronic obstructive pulmonary disease. N Eng J Med 1995; 333: 817-822.

22. Plant PK, Owen JL, Elliott MW. Early use of noninvasive ventilation for acute exacerbations of chronic obstructive pulmonary disease on general respiratory wards: a multicentre randomised controlled trial. Lancet 2000; 355: 1931-1935. 